\title{
(2) Optimal diet for cardiovascular and planetary health
}

\section{OPEN ACCESS}

Centre for Nutrition, Prevention and Health Services, National Institute for Public Health and the Environment, Bilthoven, The Netherlands

Correspondence to Professor W M Monique Verschuren, National Institute for Public Health and the Environment, Bilthoven, The Netherlands; monique.verschuren@rivm.nl

Check for updates

(c) Author(s) (or their employer(s)) 2021. Re-use permitted under CC BY-NC. No commercial re-use. See rights and permissions. Published by BMJ.

To cite: Verschuren WMM, Boer JMA, Temme EHM. Heart Epub ahead of print: [please include Day Month Year]. doi:10.1136/ heartjnl-2019-316373

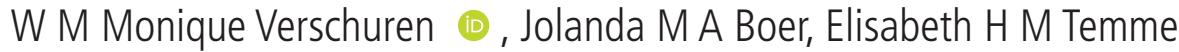

\section{INTRODUCTION}

A healthy diet is the cornerstone of cardiovascular disease (CVD) prevention. ${ }^{1}$ Dietary habits influence cardiovascular risk, through an effect on, for example, blood pressure (BP), blood lipids, obesity, inflammation and endothelial function. ${ }^{2}$ Over the past decades, knowledge about the components of a healthy diet has grown. Since there are few randomised controlled trials, most evidence on diet-disease relationships comes from prospective cohort studies, with inherent methodological problems, such as measurement error in dietary intake and correlations between dietary components. Still, the basic principles of a healthy diet can certainly be outlined. Since average dietary intake in most countries is far from optimal, the potential health gain is large, not only for CVDs but for other chronic diseases as well.

Diet also has a large impact on the environment. ${ }^{3}$ Food production systems are associated with approximately $25 \%$ of the worldwide greenhouse gas (GHG) emissions, $80 \%$ of the loss of biodiversity, $70 \%$ of water withdrawals for irrigation and $40 \%$ of land use. Therefore, dealing with environmental challenges is also a major food system challenge, especially when the foreseen growth of the world population and increasing demands for meat are considered. The EAT-Lancet commission recently introduced 'The Great Food Transformation': an urgent call for a transformation of the whole food system, from production to consumption, to decrease the total environmental impact of our current and future food system. ${ }^{4}$

In this paper, we will discuss diet in relation to both cardiovascular and planetary health.

\section{Diet and cardiovascular health}

The focus of dietary research has evolved in the past century and shifted from single nutrients to foods and subsequently to dietary patterns. ${ }^{2}$ Less than a hundred years ago, in the 1930s, vitamin C was discovered and related to the prevention of scurvy: a classic example of a 'one nutrient-one disease' situation. This started an era of studying single nutrients in relation to disease. However, we are now in the era of chronic diseases where multiple risk factors and metabolic pathways are involved. Therefore, it is easy to understand that there is no single 'magic bullet' that influences all these risk factors and pathways, and therefore, the totality of our diet plays a role. Furthermore, people eat combinations of foods, not single nutrients. Therefore, the focus has shifted to foods and dietary patterns in relation to health. We will discuss the nutrients as well as foods and dietary patterns that are most relevant and have been studied most for CVD prevention. Although focus should be on foods and dietary patterns, some background on nutrients that for long have been

\section{Learning objectives}

- This article provides information on the importance of diet for cardiovascular disease prevention. It gives insight to elements of the diet that are harmful or protective and stresses the importance of the totality of the diet. In addition, dietary choices are discussed in relation to the aspect of environmental sustainability of food production.

(and still are) a major focus in CVD prevention is useful. Estimates of the impact on CVD, coronary heart disease (CHD) or stroke risk are presented in table 1.

\section{Nutrients \\ Macronutrients}

Of the macronutrients (fat, carbohydrate and protein), dietary fatty acids have been the focus of interest since the late 1950s, due to their effect on blood lipids. The types of fatty acids consumed are important and not so much the total amount of fat in the diet. Types of fatty acids are the saturated fatty acids (SFAs; solid at room temperature) and unsaturated fatty acids (liquid at room temperature). Unsaturated fatty acids can be divided into monounsaturated fatty acids (MUFAs), polyunsaturated fatty acids (PUFAs) and trans fatty acids (TFAs). TFAs are formed mainly during industrial hydrogenation. In short-term dietary intervention studies, it has been clearly shown that SFAs increase serum total cholesterol levels, and TFAs increase serum total cholesterol and in addition lower high density lipoprotein cholesterol. However, in the majority of longitudinal cohort studies, no association of saturated fat intake with CVD has been observed. The Prospective Urban Rural Epidemiology (PURE) Study even reported a decreased mortality risk at higher intakes of SFAs. ${ }^{5}$ However, the generalisability of these results to the developed world has been questioned since the majority of the PURE study population was from low-income and middle-income countries. Many methodological issues related to observational studies play a role here, and it has become clear that when lowering SFA intake, the replacing nutrient is crucially important. Replacing SFA with PUFAs gives the largest CHD risk reduction followed by replacement with MUFAs. Replacing SFA with complex carbohydrates lowers CHD risk, while replacement with refined carbohydrates does not lower risk, and replacement with TFA increases risk. ${ }^{6}$ In conclusion, taking together the totality of evidence, avoiding TFA and replacing SFA preferably by PUFAs is recommended to lower CVD risk. The impact of dietary cholesterol on serum cholesterol levels is weak compared with that of the fatty acid composition of the diet. 
Table 1 Summary of associations of nutrients, foods and dietary patterns with cardiovascular disease risk

\begin{tabular}{|c|c|c|}
\hline Nutrients & & \\
\hline Fatty acids ${ }^{6}$ & & \\
\hline Replacing five en $\%$ SFA with PUFA & & \\
\hline Replacing five en\% SFA with MUFA & CHD & $25 \%$ lower \\
\hline Replacing five en\% SFA with complex carbohydrates & CHD & $15 \%$ lower \\
\hline Replacing SFA with refined carbohydrates & CHD & $9 \%$ lower \\
\hline Replacing SFA with TFA & CHD & None \\
\hline & CHD & $5 \%$ higher \\
\hline$\underline{\text { Minerals }}^{1112}$ & & \\
\hline 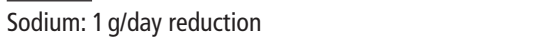 & & \\
\hline Potassium: high versus low intake ( $<120 \mathrm{mmol} / \mathrm{L})$ & CVD & $20 \%$ lower \\
\hline & Stroke & $24 \%$ lower \\
\hline Fibre $^{17}$ & & \\
\hline$\overline{7 \mathrm{~g} / \text { day }}$ higher intake & & \\
\hline $10 \mathrm{~g} /$ day higher intake & CHD & $9 \%$ lower \\
\hline $10 \mathrm{~g} /$ day higher intake & Stroke & $16 \%$ lower \\
\hline & Type 2 diabetes & $6 \%$ lower \\
\hline Food (group)s & & \\
\hline Fruits and vegetables $^{13}$ & & \\
\hline $200 \mathrm{~g} /$ day higher intake & & \\
\hline $200 \mathrm{~g} /$ day higher intake & CVD & $8 \%$ lower \\
\hline $200 \mathrm{~g} /$ day higher intake & CHD & $8 \%$ lower \\
\hline & Stroke & $16 \%$ lower \\
\hline Pulses $^{14}$ & & \\
\hline Per 4 weekly servings of $100 \mathrm{~g}$ & & \\
\hline & CHD & $14 \%$ lower \\
\hline Nuts $^{15}$ & & \\
\hline$\overline{\operatorname{Per} 28 \mathrm{~g} / \text { day }}$ & & \\
\hline Per $28 \mathrm{~g} /$ day & CVD & $21 \%$ lower \\
\hline Per $28 \mathrm{~g} /$ day & CHD & $29 \%$ lower \\
\hline & Stroke & $7 \%$ lower \\
\hline Whole grain foods ${ }^{16}$ & & \\
\hline $90 \mathrm{~g} /$ day versus no intake & & \\
\hline & CHD & $25 \%$ lower \\
\hline Red meat $^{18}$ & & \\
\hline Unprocessed: 2 versus 0 servings/week & & \\
\hline Processed: 2 versus 0 servings/week & CVD & $3 \%$ higher \\
\hline & CVD & $7 \%$ higher \\
\hline Fish $^{19}$ & & \\
\hline Per $20 \mathrm{~g} /$ day & & \\
\hline Per $20 \mathrm{~g} /$ day & CHD incidence & $4 \%$ \\
\hline & CHD mortality & $4 \%$ \\
\hline Sugar-sweetened beverages $^{23}$ & & $31 \%$ higher \\
\hline Two servings/day versus one serving/month & & \\
\hline & CVD mortality & \\
\hline Alcoholic beverages $^{25}$ & & \\
\hline Per 12.5 units/week & & \\
\hline Per 12.5 units/week & Myocardial infarction & $6 \%$ lower \\
\hline & Stroke & $14 \%$ higher \\
\hline Dietary patterns & & \\
\hline Mediterranean diet $^{2930}$ & & \\
\hline High versus low adherence & & \\
\hline Diet versus control (RCT) in high-risk individuals & CVD & $10 \%$ lower \\
\hline & CVD & $31 \%$ lower \\
\hline${\underline{\text { DASH }} \operatorname{diet}^{22}}^{2}$ & & \\
\hline High versus low adherence & & \\
\hline High versus low adherence & CVD & $20 \%$ lower \\
\hline High versus low adherence & CHD & $20 \%$ lower \\
\hline & Stroke & $20 \%$ lower \\
\hline
\end{tabular}

$\mathrm{CHD}$, coronary heart disease; $\mathrm{CVD}$, cardiovascular disease; DASH, dietary approaches to stop hypertension; En\%, percentage of total energy intake; MUFA, monounsaturated fatty acid; PUFA, polyunsaturated fatty acid; RCT, randomised controlled trial; SFA, saturated fatty acid; TFA, trans fatty acid.

The intake of carbohydrates and proteins in relation to CVD has been mostly addressed in studies on low-carb/high-protein diets. These diets are characterised-as the name already conveys-by a (very) low intake of carbohydrates and a high intake of proteins. A very low carb diet (ketogenic diet) has been shown to contribute to glycaemic and lipid control and contributes significantly to weight loss in patients with type 2 diabetes. ${ }^{7}$ However, when considering long-term effects, there is currently insufficient evidence to recommend such diets to improve cardiovascular health. They may even be associated with increased all-cause mortality when used for extended periods. ${ }^{8}$ With respect to carbohydrates, we distinguish simple carbohydrates (monosaccharides and disaccharides, sugars) and complex carbohydrates (polysaccharides, starch). Refined carbohydrates are often depleted of their vitamin, mineral and fibre content. Unrefined complex carbohydrates take longer to break down and therefore impact blood glucose level more slowly, which is important for cardiovascular health.

\section{Vitamins and minerals}

Sodium and potassium are the most important minerals for CVD prevention due to their effect on BP. ${ }^{9}$ Although the relation between sodium (table salt) intake and BP has recently been questioned, ${ }^{10}$ the totality of evidence still warrants sodium reduction as an important way to prevent CVD. ${ }^{11}$ In most Western countries, sodium intake is high (around $3.6-4 \mathrm{~g} /$ day corresponding to $9-10 \mathrm{~g}$ of salt), whereas the recommended maximum intake is $2 \mathrm{~g} /$ day (5 g/day salt) and optimal intake levels might be as low as around $1.2 \mathrm{~g} /$ day ( $3 \mathrm{~g} /$ day salt). Potassium has favourable effects on BP, and a higher intake of potassium has been shown to reduce the risk of incident stroke. ${ }^{12}$ Main sources of potassium are fruits, vegetables, whole grains and meat.

Evidence for a protective effect of vitamins (vitamin A, B6, B12, C, D, E and folic acid) is not yet conclusive.

\section{Foods and food groups}

Plant-based foods

Prospective cohort studies have shown a protective effect of consumption of fruits and vegetables on CVD, with risk decreasing up to an intake of 800 g per day. ${ }^{13}$ A meta-analysis of cohort studies showed that consumption of pulses is associated with a reduced CHD risk, but there was no association with stroke or type 2 diabetes. ${ }^{14}$ Pulses have a low glycaemic index, are low in fat and high in fibre, protein, B vitamins and minerals such as iron, calcium and potassium. These aspects likely contribute to their observed health effect. Also the consumption of nuts has been shown to reduce CVD risk. ${ }^{15}$ It must be noted, however, that the energy density of nuts is high, increasing the risk of overweight when consumed in high quantities. Incorporating nuts as part of the diet, for example, to replace meat, can help to maintain energy balance. Consumption of whole grain foods has been shown to reduce the risk of CHD. ${ }^{16}$ Whole grains are rich in nutrients, phytochemicals such as antioxidants, 
Table 2 Comparing the mean Dutch dietary consumption (2012-2016) to the recommended intake by the Dutch dietary guidelines and the 'healthy reference diet' proposed by Willett et al ${ }^{435} 36$

\begin{tabular}{llll}
\hline $\begin{array}{l}\text { Food group } \\
\text { (g/day) }\end{array}$ & $\begin{array}{l}\text { Healthy reference } \\
\text { diet }^{4}\end{array}$ & $\begin{array}{l}\text { Dutch healthy diet } \\
\text { guidelines*35 }\end{array}$ & $\begin{array}{l}\text { Dutch National Food } \\
\text { Consumption Survey }\end{array}$ \\
\hline $\begin{array}{l}\text { Whole grain foods } \\
\text { Potatoes }\end{array}$ & 232 & $\geq 90$ & 93 \\
Vegetables & $50(0-100)$ & - & 72 \\
Fruit & $300(200-600)$ & $\geq 200$ & 143 \\
Dairy & $200(100-300)$ & $\geq 200$ & 113 \\
Meat & $250(0-500)$ & $300-450^{*}$ & 333 \\
& $\leq 43 \dagger(0-86)$ & $<45$ (red meat) $^{*}$ & 98 \\
Eggs & $13(0-25)$ & 0 (processed meat) $^{36}$ & - \\
Fish & $28(0-100)$ & 15 & 13 \\
Legumes & $75(0-100)$ & 10 & 5 \\
Nuts & $50(0-75)$ & $\geq 15$ & 9 \\
\hline *When the Dutch dietary guidelines do not quantify a recommendation, the cut-off values were based
\end{tabular}

*When the Dutch dietary guidelines do not quantify a recommendation, the cut-off values were based on those defined for the Dutch Healthy Diet index 2015 (DHD15 index), which is based on the Dutch dietary guidelines and the Wheel of Five.

tMainly chicken/poultry and very little red meat.

vitamins and minerals and dietary fibre. Prospective cohort studies show that higher intake of fibre is associated with lower risk of CHD, stroke and type 2 diabetes. ${ }^{17}$ Mechanisms may include low density lipoprotein cholesterol lowering, immune protection and improvement in glucose metabolism and may differ per type of grain.

For most environmental aspects (eg, GHG emission and land use), plant-based foods have a low impact. However, fresh water use may be high for nuts and some types of fruits, such as oranges especially when grown in water scarce regions. ${ }^{4}$

\section{Animal-based foods}

Higher intake of red meat, and especially processed meat, has been associated with a higher risk of CVD. ${ }^{18}$ Red and processed meats are a source of saturated fatty acids, whereas processed meat may also be high in sodium. Several potential

\section{Box 1 Current dietary recommendations ${ }^{1783537}$}

Based on all evidence available, in general, the following recommendations are given by current guidelines for a healthy diet:

- Saturated fatty acid intake accounts for less than $10 \%$ of total energy intake, through replacement by polyunsaturated fatty acids.

- Trans unsaturated fatty acids are consumed as little as possible, preferably no intake from processed food and $<1 \%$ of total energy intake from natural trans fatty acid origins.

$<5 \mathrm{~g}$ of salt per day.

- 30-45 g of fibre per day, preferably from whole grain products.

- $\geq 200 \mathrm{~g}$ of fruit per day (2-3 servings).

- $\geq 200 \mathrm{~g}$ of vegetables per day (2-3 servings).

- Fish once a week, preferably oily fish.

- Alcohol consumption limited to 1 glass per day (maximum ethanol intake of $100 \mathrm{~g} /$ week).

- Sugar-sweetened soft drinks are discouraged; sugar should be less than $10 \%$ of total energy intake.

- 15-30 g unsalted nuts per day.

- Shift towards a plant-based diet, less red and processed meat. mechanisms by which the consumption of red and processed meat may increase the risk of disease have been postulated, but conclusive evidence is lacking. Of all foods, red meat has per kilogram the highest negative impact on the environment for GHG emissions and land use, followed by other animal-based foods like cheese and fish. Results from prospective cohort studies show that compared with no fish intake, fish intake is associated with a lower CHD incidence and lower CHD mortality ${ }^{19}$ but not with incident CVD. ${ }^{18}$ The largest risk reduction is obtained when people increase their intake from no fish or very little fish to eating fish once a week. The protective effect of fish on CHD is attributed to its n-3 fatty acid content; therefore, oily fish is recommended once a week. Fish oil supplements do not lower overall cardiovascular risk, although there may be a slight beneficial effect on the risk of a CHD event and CHD mortality. ${ }^{20}$ From an environmental perspective, overfishing of wild fish is a major issue for biodiversity loss with about $60 \%$ of the world fish stocks fully fished and more than $30 \%$ overfished. $^{4}$

For (low-fat) dairy, there is no conclusive evidence that it is associated with increased CVD risk. ${ }^{8} 2122$ In current dietary patterns, dairy (including cheese) is a significant contributor to its environmental impact.

\section{Other}

Sugar-sweetened beverages (SSBs) contribute to caloric intake but contain little or no nutrients. Regular consumption of SSBs has been associated with overweight, metabolic syndrome, type 2 diabetes and a higher risk of CVD mortality. ${ }^{23} 24$ Based on studies on SSBs, WHO recommends a maximum intake of $10 \%$ of energy from sugar (monosaccharides and disaccharides) that includes added sugars as well as sugars naturally present in beverages, fruits and fruit juices. With respect to alcoholic beverages, a large meta-analysis concluded that the association between consumption of alcoholic beverages and total CVD is actually comprised of several distinct and opposite dose-response curves rather than a single J-shaped curve. ${ }^{25}$ For stroke, risk increased linearly, while for myocardial infarction, risk decreased with increasing alcohol consumption. For the composite endpoint of all CVD, the lowest risk was observed at an alcohol intake level of $100 \mathrm{~g}$ pure alcohol per week corresponding to for example or approximately seven glasses of wine per week. Alcoholic drinks, although plant based, like SSB, have a rather small environmental impact, for example, low GHG emission and land use, but as there are no clear health benefits, these environmental impacts could be avoided.

\section{Dietary patterns}

As mentioned earlier, there are no 'magic bullets' or 'forbidden fruits'. It is the totality of the diet that matters, because foods can have synergistic effects, and a varied diet influences multiple metabolic pathways. Not only the quality of the diet is important, but given the fact that obesity is a growing problem, also quantity 
is important. A healthy diet contributes to obtaining or maintaining a healthy body weight. Guiding principles for a healthy diet are that it is mainly plant based, fresh and as minimally processed as possible, with no or moderate alcohol intake. It is important to avoid ultraprocessed foods (industrially manufactured foods) because these foods contain less nutrients and more energy than fresh foods because they are depleted from fibre and micronutrients, while containing more added sugar, salt and/or fat. ${ }^{26}$ Following these principles will automatically lead to a diet low in salt, sugar and industrial fat, and high in fibre, which in turn will contribute to obtaining or maintaining a healthy weight and cardiovascular health. Usually, a plant-based diet also implies a low environmental impact because the environmental impact of plant-based foods is in general lower than that of animal based foods. ${ }^{27} 28$ We will briefly discuss some dietary patterns that have become examples of diets that incorporate these guiding principles.

The best-known example of a healthy dietary pattern is the traditional Mediterranean diet. It comprises many of the nutrients and foods that have been discussed previously: high intake of fruits, vegetables, pulses, wholegrains, fish and unsaturated fatty acids (especially olive oil), moderate alcohol consumption and a low consumption of meat (especially red and processed), dairy and saturated fatty acids. Greater adherence to the Mediterranean diet has been associated with a reduction in CVD incidence or mortality in prospective cohort studies and an RCT in high-risk individuals. ${ }^{29} 30$ However, a recent Cochrane review rates the quality of evidence for primary prevention as low to moderate and mentions the paucity of evidence for secondary prevention. Another example of a healthy dietary pattern is the DASH diet. It was specifically designed to lower BP in the Dietary Approaches to Stop Hypertension Trial. This diet is rich in fruits, vegetables, whole grains and low-fat dairy, and low

\section{Key messages}

- Diet is the cornerstone of cardiovascular disease prevention: it influences a great number of risk factors and metabolic pathways and is important for weight maintenance.

- Recommendations of current dietary guidelines are summarised in Box 1

- Focus should be on healthy foods and dietary patterns, not on single nutrients (there is no magic bullet).

- A guiding principle in making healthy dietary choices is to shift towards a plant-based diet and avoid energy dense ultra-processed foods rich in salt, sugar and fat. Key components of a healthy diet are: fruits, vegetables, pulses, nuts, whole grains, fish, low fat dairy and vegetable oils.

- A plant-based diet with limited amounts of animal-based foods, using local and seasonal products, is good for human and planetary health.

- Reduction of food waste contributes to reducing environmental impact.

- Dietary changes are not just the responsibility of the individual. Societal and policy measures can greatly contribute to making the healthy choice the easy choice, by improving our food environment. Industry can contribute by reformulating ultra-processed foods (less sugar, salt, saturated and trans fatty acids).

- Current diets are still far from the optimal diet, so the potential health gain is large for both people and planet. in saturated fat, salt, sugar and red and processed meats. It has been associated with a reduction in BP and decreases in CVD, CHD and stroke. ${ }^{22}$ The Nordic dietary pattern is characterised by a high intake of fish, whole grains including oats, rye and barley and specific fruit and vegetables: apples/pears, berries, root vegetables and cabbages. Intervention studies have shown various short-term beneficial effects of the Nordic dietary pattern among individuals with increased CVD risk. However, there is no convincing evidence yet for an association between the Nordic dietary pattern and CVD. ${ }^{22}$ The Mediterranean, DASH and Nordic dietary patterns are all high in plant-based foods and low in animal-based foods and have a lower environmental impact than most current diets. ${ }^{31}$ From a sustainability point of view, it is furthermore important to use seasonal products, preferably locally grown, and to avoid waste. The Nordic and Mediterranean diets are good examples of diets that are adapted to the local situation of the Nordic respectively Mediterranean countries, with respect to the availability of foods and eating habits. A global 'healthy reference diet' for healthier people around the globe and a healthy planet with even higher consumption of whole grains, pulses and nuts has been suggested by the EAT Lancet commission (see table 2 ). ${ }^{4}$

\section{Changing our diet}

Changing long-standing dietary habits has proven very difficult. Current Western diets, such as the Dutch diet, are far from current recommendations and the recently proposed 'healthy reference diet' (table 2). For example, total meat consumption is twice the recommend quantity, whereas pulses and nuts consumption are far below both recommendations. It will take time and a shift of the norm to make the required major modifications to the current diet and to achieve such a healthy and sustainable diet. We have simulated the environmental impact of changing the current Dutch diet to a diet recommended by dietary guidelines. When adhering to the dietary guideline, it depends on the dietary choices made to what extent the environmental impact is reduced. With omitting meat or choosing foods with relatively low associated GHG emissions, it is possible to reduce GHG emission by about a third compared with current Dutch diets. ${ }^{32}$

Diet is more than eating recommended amounts of nutrients and foods, since diet also has a social and cultural meaning. It is also important to realise that for people with a low socioeconomic position, financial aspects can be a barrier for adopting a healthier and more sustainable diet. Fresh fruits and vegetables are often relatively expensive. Furthermore, changing dietary habits is not only an individual challenge, but a challenge for our society. Our food environment stimulates overconsumption, causing the current obesity epidemic and contributing to our ecological footprint. Policy measures, such as a ban on advertisements and taxation of unhealthy foods or subsidising healthy foods, will help people to make more healthy and sustainable choices. ${ }^{33}$ Food industry can have 
CME credits for Education in Heart

Education in Heart articles are accredited for CME by various providers. To answer the accompanying multiple choice questions (MCQs) and obtain your credits, click on the 'Take the Test' link on the online version of the article. The MCQs are hosted on BMJ Learning. All users must complete a one-time registration on BMJ Learning and subsequently log in on every visit using their username and password to access modules and their CME record. Accreditation is only valid for 2 years from the date of publication. Printable CME certificates are available to users that achieve the minimum pass mark.

an important contribution by lowering salt, sugar and (trans-)saturated fat content of products (food reformulation). A good example is lowering the salt content of many products by the food industry, and the almost total elimination of trans fatty acids. In this way, people eat healthier diets without having to change their dietary habits. For reducing environmental impact, changes throughout the food production chain are needed. ${ }^{4}$

In summary, people have to be educated and supported in changing their lifestyle, so that the healthy and environmentally friendly choice will become the easy choice for them. In addition, a global transformation of the food system is needed to reduce the environmental impact of our diet.

Contributors JMAB and EHMT contributed substantially to the manuscript.

Funding The authors have not declared a specific grant for this research from any funding agency in the public, commercial or not-for-profit sectors.

Competing interests None declared.

Patient and public involvement Patients and/or the public were not involved in the design, or conduct, or reporting, or dissemination plans of this research.

Patient consent for publication Not applicable.

Provenance and peer review Commissioned; externally peer reviewed.

Open access This is an open access article distributed in accordance with the Creative Commons Attribution Non Commercial (CC BY-NC 4.0) license, which permits others to distribute, remix, adapt, build upon this work non-commercially, and license their derivative works on different terms, provided the original work is properly cited, appropriate credit is given, any changes made indicated, and the use is non-commercial. See: http:// creativecommons.org/licenses/by-nc/4.0/.

\section{ORCID iD}

W M Monique Verschuren http://orcid.org/0000-0003-2134-4227

\section{REFERENCES}

1 European Heart Network. Transforming European food and drink policies for cardiovascular health. Brussels: European Heart Network, 2017.

2 Mozaffarian D. Dietary and policy priorities for cardiovascular disease, diabetes, and obesity: a comprehensive review. Circulation 2016;133:187-225.

3 ed.: .Birt C, Buzeti T, Grosso G. Healthy and Sustainable diets for European countries. In: EUPHA. Brussels: European Public Health Association - EUPHA, 2017.

4 Willett W, Rockström J, Loken B, et al. Food in the Anthropocene: the EAT-Lancet Commission on healthy diets from sustainable food systems. Lancet 2019;393:447-92.

5 Dehghan M, Mente A, Zhang $X$, et al. Associations of fats and carbohydrate intake with cardiovascular disease and mortality in 18 countries from five continents (pure): a prospective cohort study. Lancet 2017;390:2050-62.

6 Li Y, Hruby A, Bernstein AM, et al. Saturated Fats Compared With Unsaturated Fats and Sources of Carbohydrates in Relation to Risk of Coronary Heart Disease: A Prospective Cohort Study. J Am Coll Cardiol 2015:66:1538-48

7 Yuan X, Wang J, Yang S, et al. Effect of the ketogenic diet on glycemic control, insulin resistance, and lipid metabolism in patients with T2DM: a systematic review and meta-analysis. Nutr Diabetes 2020;10:38.

8 Pallazola VA, Davis DM, Whelton SP, et al. A clinician's guide to healthy eating for cardiovascular disease prevention. Mayo Clin Proc Innov Qual Outcomes 2019;3:251-67.

9 Sacks FM, Svetkey LP, Vollmer WM, et al. Effects on blood pressure of reduced dietary sodium and the dietary approaches to stop hypertension (DASH) diet. DASH-Sodium Collaborative Research Group. N Engl J Med 2001;344:3-10.

10 Mente A, O'Donnell M, Rangarajan S, et al. Urinary sodium excretion, blood pressure, cardiovascular disease, and mortality: a community-level prospective epidemiological cohort study. Lancet 2018:392:496-506.

11 He FJ, Tan M, Ma Y, et al. Salt reduction to prevent hypertension and cardiovascular disease: JACC state-of-the-art review. J Am Coll Cardiol 2020;75:632-47.

12 Aburto NJ, Hanson S, Gutierrez H, et al. Effect of increased potassium intake on cardiovascular risk factors and disease: systematic review and meta-analyses. BMJ 2013;346:f1378.

13 Aune D, Giovannucci E, Boffetta P, et al. Fruit and vegetable intake and the risk of cardiovascular disease, total cancer and all-cause mortality-a systematic review and dose-response meta-analysis of prospective studies. Int J Epidemiol 2017;46:1029-56.

14 Afshin A, Micha R, Khatibzadeh S, et al. Consumption of nuts and legumes and risk of incident ischemic heart disease, stroke, and diabetes: a systematic review and meta-analysis. Am J Clin Nutr 2014;100:278-88

15 Aune D, Keum N, Giovannucci E, et al. Nut consumption and risk of cardiovascular disease, total cancer, all-cause and cause-specific mortality: a systematic review and dose-response meta-analysis of prospective studies. BMC Med 2016:14:207.

16 Anderson JW, Hanna TJ, Peng $X$, et al. Whole grain foods and heart disease risk. J Am Coll Nutr 2000;19:291S-9.

17 Threapleton DE, Greenwood DC, Evans CEL, et al. Dietary fibre intake and risk of cardiovascular disease: systematic review and meta-analysis. BMJ 2013;347:f6879.

18 Zhong VW, Van Horn L, Greenland P, et al. Associations of processed meat, unprocessed red meat, poultry, or fish intake with incident cardiovascular disease and all-cause mortality. JAMA Intern Med 2020;180:503-12.

19 Zhang B, Xiong K, Cai J, et al. Fish consumption and coronary heart disease: a meta-analysis. Nutrients 2020;12:2278.

20 Abdelhamid AS, Brown TJ, Brainard JS, et al. Omega-3 fatty acids for the primary and secondary prevention of cardiovascular disease. Cochrane Database Syst Rev 2020;3:CD003177.

21 Bhupathi V, Mazariegos M, Cruz Rodriguez JB, et al. Dairy intake and risk of cardiovascular disease. Curr Cardiol Rep 2020;22:11.

22 Zampelas A, Magriplis E. Dietary patterns and risk of cardiovascular diseases: a review of the evidence. Proc Nutr Soc 2020;79:68-75.

23 Malik VS, Li Y, Pan A, et al. Long-Term consumption of sugarsweetened and artificially sweetened beverages and risk of mortality in US adults. Circulation 2019;139:2113-25.

24 Tappy L, Morio B, Azzout-Marniche D, et al. French recommendations for sugar intake in adults: a novel approach chosen by ANSES. Nutrients 2018:10:989.

25 Wood AM, Kaptoge S, Butterworth AS, et al. Risk thresholds for alcohol consumption: combined analysis of individual-participant data for 599912 current drinkers in 83 prospective studies. Lancet 2018:391:1513-23.

26 Monteiro CA, Cannon G, Lawrence M. Ultra-processed foods, diet quality, and health using the nova classification system. Rome: FAO, 2019.

27 Clark MA, Springmann M, Hill J, et al. Multiple health and environmental impacts of foods. Proc Natl Acad Sci U SA 2019;116:23357-62.

28 Grosso G, Fresán U, Bes-Rastrollo M, et al. Environmental impact of dietary choices: role of the Mediterranean and other dietary patterns in an Italian cohort. Int J Environ Res Public Health 2020;17:1468. 
29 Sofi F, Abbate R, Gensini GF, et al. Accruing evidence on benefits of adherence to the Mediterranean diet on health: an updated systematic review and meta-analysis. Am J Clin Nutr 2010;92:1189-96.

30 Estruch R, Ros E, Salas-Salvadó J, et al. Primary prevention of cardiovascular disease with a Mediterranean diet supplemented with extra-virgin olive oil or nuts. N Engl J Med 2018:378:e34.

31 Nelson ME, Hamm MW, Hu FB, et al. Alignment of healthy dietary patterns and environmental sustainability: a systematic review. $A d v$ Nutr 2016:7:1005-25.

32 van de Kamp ME, van Dooren C, Hollander A, et al. Healthy diets with reduced environmental impact? - The greenhouse gas emissions of various diets adhering to the Dutch food based dietary guidelines. Food Res Int 2018;104:14-24.
33 Afshin A, Peñalvo JL, Del Gobbo L, et al. The prospective impact of food pricing on improving dietary consumption: a systematic review and meta-analysis. PLoS One 2017;12 e0172277.

34 WHO. European food and nutrition action plan 2015-2020. Copenhagen: WHO Rgeional Office for Europe, 2015.

35 Kromhout D, Spaaij CJK, de Goede J, et al. The 2015 Dutch food-based dietary guidelines. Eur J Clin Nutr 2016;70:869-78.

36 RIVM. Wat EET Nederland. Bilthoven: RIVM, 2018.

37 U.S. Department of Health and Human Services and U.S. Department of Agriculture. 2015-2020 dietary guidelines for Americans 2015 